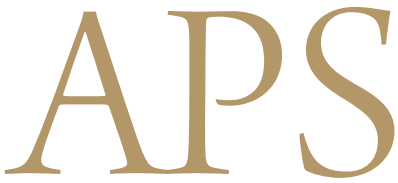

Archives of Plastic Surgery

\title{
Tarsodermal Suture Fixation Preceding Redundant Skin Excision: A Modified Non-Incisional Upper Blepharoplasty Method for Elderly Patients
}

\author{
Hong Sang Yoon ${ }^{1}$, Bo Young Park ${ }^{2}$, Kap Sung $\mathrm{Oh}^{1}$ \\ ${ }^{1}$ Department of Plastic Surgery, Samsung Medical Center, Sungkyunkwan University School of Medicine, Seoul; ${ }^{2}$ Department of Plastic \\ Surgery, Ewha Womans University School of Medicine, Seoul, Korea
}

Background Non-incisional blepharoplasty is a simple, less invasive method for creating a more natural-appearing double eyelid than classical incisional blepharoplasty. However, in aging patients, non-incisional blepharoplasty is not effective due to more severe blepharochalasis. Traditionally, incisional blepharoplasty is a common surgical method used for older patients, but blepharoplasty in elderly patients typically results in prolonged recovery times, and final blepharoplasty lines may be located in unintended or asymmetrical positions. Here, we introduce a new modified combination technique for geriatric blepharoplasty.

Methods A total of ten patients were treated from July 2010 through July 2012 using the combination method. First, we performed non-incisional blepharoplasty using tarsodermal fixation. Then, incisional blepharoplasty with additional elliptical excision of the upper eyelid skin was performed. We removed pretarsal tissue, fat, the orbicularis oculi muscle, and orbital fat. Telephone surveys were administered to all patients for follow-up. The questionnaire was composed of eight questions that addressed recurrence and satisfaction with aesthetics and the procedure.

Results A total of nine patients (90\%) responded to the telephone survey. All cases of moderate to severe blepharochalasia were corrected and there were no major complications. Patients who underwent blepharoplasty had higher satisfaction scores. All patients were satisfied with the postoperative shapes of their eyelids.

Conclusions The advantages of the proposed technique include: ease of obtaining a naturallooking fold with symmetry at the desired point; reproducible methods that require short operation times; fast postoperative recovery that results in a natural-appearing double-eyelid line; and high patient satisfaction.

Keywords Blepharoplasty / Blepharoptosis / Eyelids
Correspondence: Kap Sung Oh Department of Plastic Surgery, Samsung Medical Center,

Sungkyunkwan University School of Medicine, 81 Irwon-ro, Gangnam-gu, Seoul 135-710, Korea

Tel: $+82-2-3410-2210$

Fax: +82-2-3410-0036

E-mail: kapsung.oh@samsung.com

No potential conflict of interest relevant to this article was reported.

\section{INTRODUCTION}

Double-eyelid operations are the most commonly performed aesthetic surgical procedures in Korea, and the skills and techniques required for the operation have developed over time. Traditionally, incisional blepharoplasty was the most common 
surgical method, but non-incisional blepharoplasty is being performed more frequently due to the desire to minimize scarring and decrease postoperative complications [1]. Because of patient demand for natural-looking folds, short operative times, minimal scarring, and fast postsurgical recovery, procedures are changing from full incision to non-incisional methods.

Although non-incisional blepharoplasty typically results in a natural-looking appearance and fast postoperative recovery, elderly patients commonly have redundant skin and sunken eyelids that limit the efficacy of the procedure compared to younger patients. Furthermore, blepharoplasty in elderly patients typically results in prolonged recovery times, and final blepharoplasty lines may be located in unintended or asymmetrical positions as redundant skin interferes with accurate blepharoplasty lines.

We present a new combination technique using a non-incisional method to produce natural-looking blepharoplasty lines at precise, intended positions in elderly patients. Our modified upper blepharoplasty technique is expected to provide improved aesthetic results, particularly in elderly patients.

\section{METHODS}

A total of 10 patients were treated from July 2010 through July 2012. Two patients were male and the remaining eight patients were female. The mean age of the patients was 60.1 years (Table

\begin{tabular}{|c|c|}
\hline Characteristic & n (\%) \\
\hline \multicolumn{2}{|l|}{ Sex } \\
\hline Male & $2(20)$ \\
\hline Female & $8(80)$ \\
\hline Total & $10(100)$ \\
\hline \multicolumn{2}{|l|}{ Age (yr) } \\
\hline 40 to 60 & $5(50)$ \\
\hline 61 to 70 & $4(40)$ \\
\hline 71 to 80 & $1(10)$ \\
\hline Total & $10(100)$ \\
\hline Mean age (yr) & 60.1 \\
\hline
\end{tabular}

1). Precise preoperative observations of both eyes were performed initially to determine the locations of the double-eyelid lines and the appropriate dimensions. Prior to surgery, with the patient in a sitting position and both eyes level, we pressed at the midpupillary point with forceps to demonstrate the potential future line in order to select the position of the fold with input from the patient. The lower line was designed first, as this is the line where the tarsal plate is fixed to make a double fold at 6-8 $\mathrm{mm}$ above the ciliary margin. Three points were marked with a marking pencil on the line of the double eyelid while the patient was seated ( $a, b$, and $c)$. Thereafter, an additional three points (d, e, and f) were marked 2-3 mm medially from the previous three points ( $a, b$, and $c$ ). Point $d$ was near the medial canthus, and point $e$ was at the middle one third of the upper eyelid. Point $f$ was near the lateral canthus (Fig. 1A) [2,3].

After the line for the double eyelid was determined, we selected the location of the incision and suture. The lower line of the skin design for excision was marked about $2 \mathrm{~mm}$ above the tarsodermal fixation line because when the line is less than $2 \mathrm{~mm}$ above the tarsodermal fixation line, wound closure of the skin excision site is typically more difficult and when the line is more than $2 \mathrm{~mm}$ above the tarsodermal fixation line, two double fold lines may be formed. The width of the skin excision was about 4-8 $\mathrm{mm}$ at the midpupillary point, $2.5 \mathrm{~mm}$ at the lateral canthal area, and $1 \mathrm{~mm}$ at the medial canthus area (Fig. 1B).

A small amount of $2 \%$ lidocaine containing 1:100,000 epinephrine was infiltrated into the subcutaneous layer and the tarsal plate at the surgical site. Tagging sutures were placed in the upper eyelid using black silk 6-0 suture material. Three puncture wounds 2-3 mm long were made along the preoperative markings with a No. 11 blade after everting the upper eyelid using a cotton swab. Double-armed suture needles with 7-0 nylon were penetrated through the tarsal plate from the conjunctival surface to the eyelid skin through each incision. Two buried interstitch ligations were performed by passing one of the double-armed needles with thread subcutaneously from one puncture wound to another. In the medial and lateral puncture wounds, single su-

\section{Fig. 1. Surgical design for the combination method}

(A) Three points $(a, b$, and $c)$ were marked at 6-8 $\mathrm{mm}$ above the ciliary margin on the line of the double eyelid while the patient was seated. Three points ( $d, e$ and f) were marked 2-3 $\mathrm{mm}$ medially from the previous three points $(a, b$ and $c)$. Point $d$ was near the medial canthus, point e was at the middle one-third of the upper eyelid. Point $f$ was near the lateral canthus (B). We made three slit incisions of 2-3 $\mathrm{mm}$ length with a No. 11 blade. The blue line indicates the skin design for excision marked about $5 \mathrm{~mm}$ above the tarsodermal fixation line $(A, B)$.

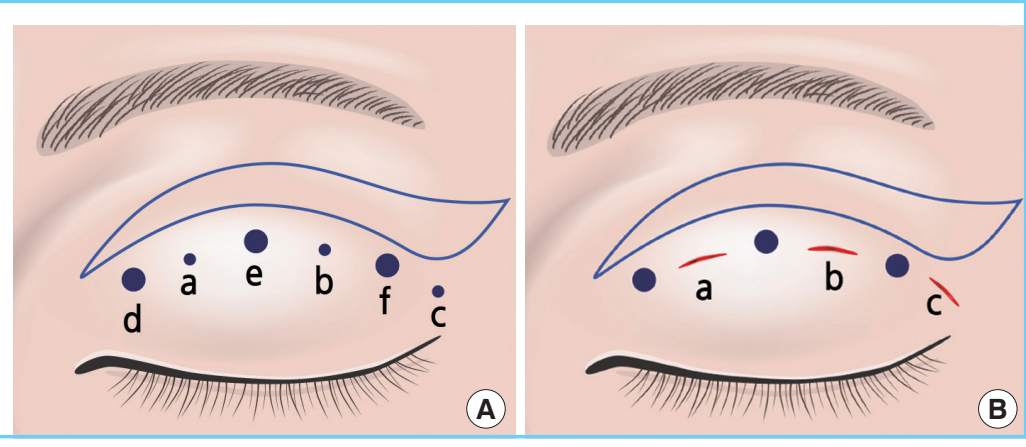


ture needles split from double-armed suture needles with 7-0 nylon were used to make the double fold (Fig. 2). If the surgeon needed to remove fat tissue, a small amount of $2 \%$ lidocaine containing 1:100,000 epinephrine was infiltrated into the underlying fat. Small forceps were then used to grasp part of the orbital fat from the lateral side. After the orbital septum was opened, the yellowish fat pad could be removed with soft palpation of the upper eyelid, and an elliptical skin incision was made using a no. 15 blade according to the previous skin incision design. The pretarsal orbicularis oculi muscle was preserved in most cases. The same procedure was used on the contralateral eye. The skin incision was closed with one $7 / 0$ black silk stitch.

In the immediate postoperative period, antibiotic eye ointment was applied over the upper eyelid and the patient was instructed to compress the upper eyelid. Patients were discharged on the same day and asked to apply an ice pack to the surgical site for the first day. Oral antibiotics were administered for three days. Partial stitch removal from the slit incision site was performed on the third or fourth day after surgery. Complete suture removal from the skin excision site was accomplished on the seventh day at the second clinic visit. Patients were instructed not to rub their eyelids for a week.

Surveys were administered to all patients who underwent blepharoplasty using our modified method. The questionnaire was composed of eight questions that addressed recurrence and satisfaction with aesthetics and procedure.

\section{Fig. 2. Tarsodermal fixation procedure similar to non-} incisional blepharoplasty

With the upper eyelid everted, double-armed suture needles with 7-0 nylon were penetrated through the tarsal plate from the conjunctival surface to the eyelid skin through each incision above the superior tarsal border. Two buried interstitch ligations were performed by passing one of the double-armed needles with thread subcutaneously from one puncture wound to another. The same procedure was repeated through all three mini-incisions.

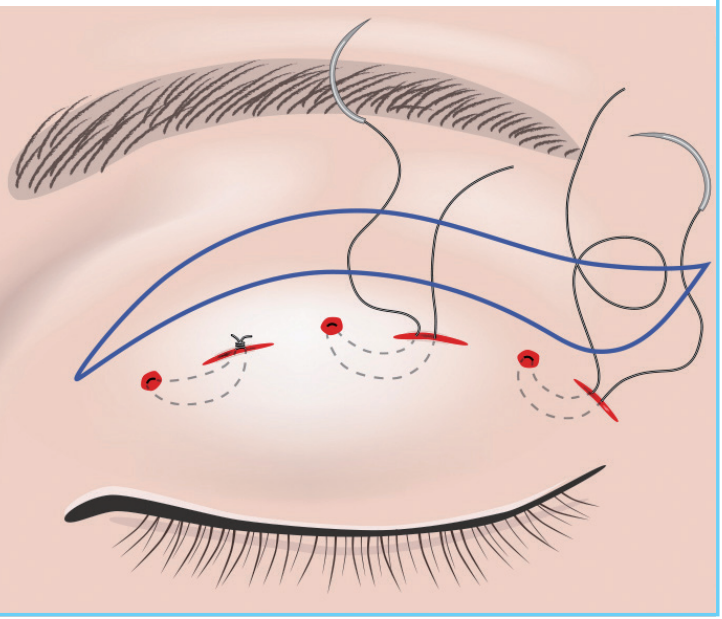

\section{RESULTS}

All cases of moderate to severe blepharochalasia were corrected and there were no major complications including hematoma, granuloma, dry-eye syndrome, infection, or exposure of the suture material. There were instances of mild swelling or bruising for a few days after surgery.

A total of nine patients (90\%) responded to the telephone survey. Patients who underwent blepharoplasty had higher satisfaction scores (Table 2). All patients were satisfied with the postoperative shapes of their eyelids. Some patients complained about their immediate postoperative results, but they were satisfied with the results in the postoperative follow-up period (Figs. $3,4)$. There were no complaints of asymmetry or loosening of the double eyelids.

\begin{tabular}{|lc|}
\hline \multicolumn{2}{|l|}{ Table 2. Postoperative survey of patient satisfaction } \\
\hline Characteristic & Value \\
\hline Survey response rate & $9 / 10(90 \%)$ \\
& Score $(-2$ to +2$)$ \\
Satisfaction for ptosis & 0.6 \\
Satisfaction for pain & 1 \\
Satisfaction for symmetry & 1.2 \\
Satisfaction for aesthetics & 1 \\
Satisfaction for scar & 2 \\
Satisfaction for recurrence & 0.8 \\
Experience for complications & 2 \\
Satisfaction for procedure & 1 \\
\hline
\end{tabular}

\section{Fig. 3. Case of a 55-year-old woman}

(A) Photographs show a 55-year-old woman with moderate ptosis and redundant skin. (B) Photographs were taken two months after surgery. (C) Photographs of the closed eye at postoperative two month follow-up.
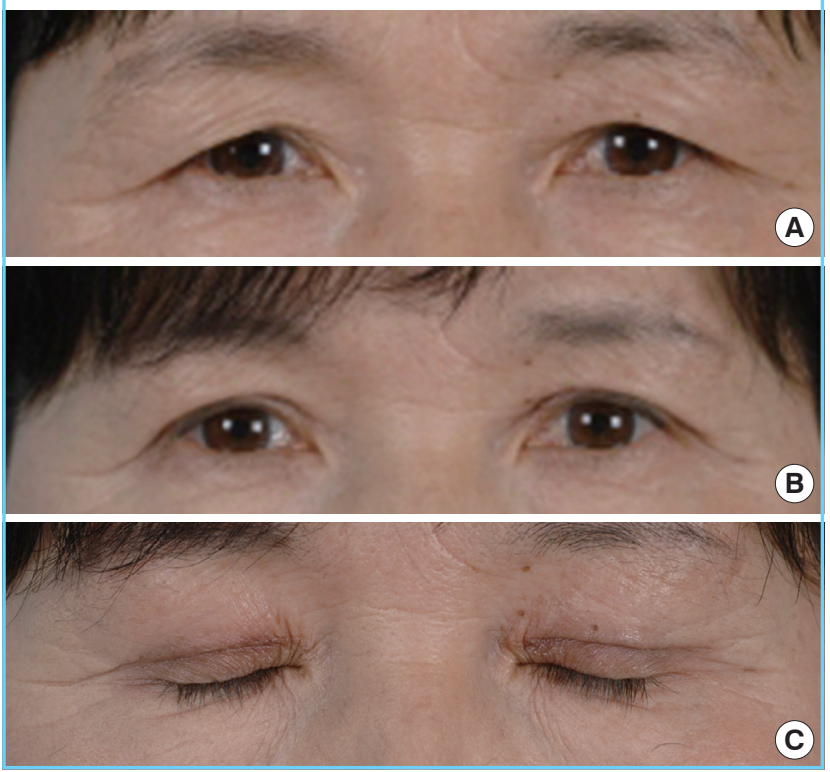


\section{Fig. 4. Case of a 64-year-old woman}

(A) Photographs show a 64-year-old woman with severe ptosis and redundant skin preoperatively, (B) two months, and (C) six months postoperatively. (D) Photographs of the closed eye at postoperative six month follow-up.
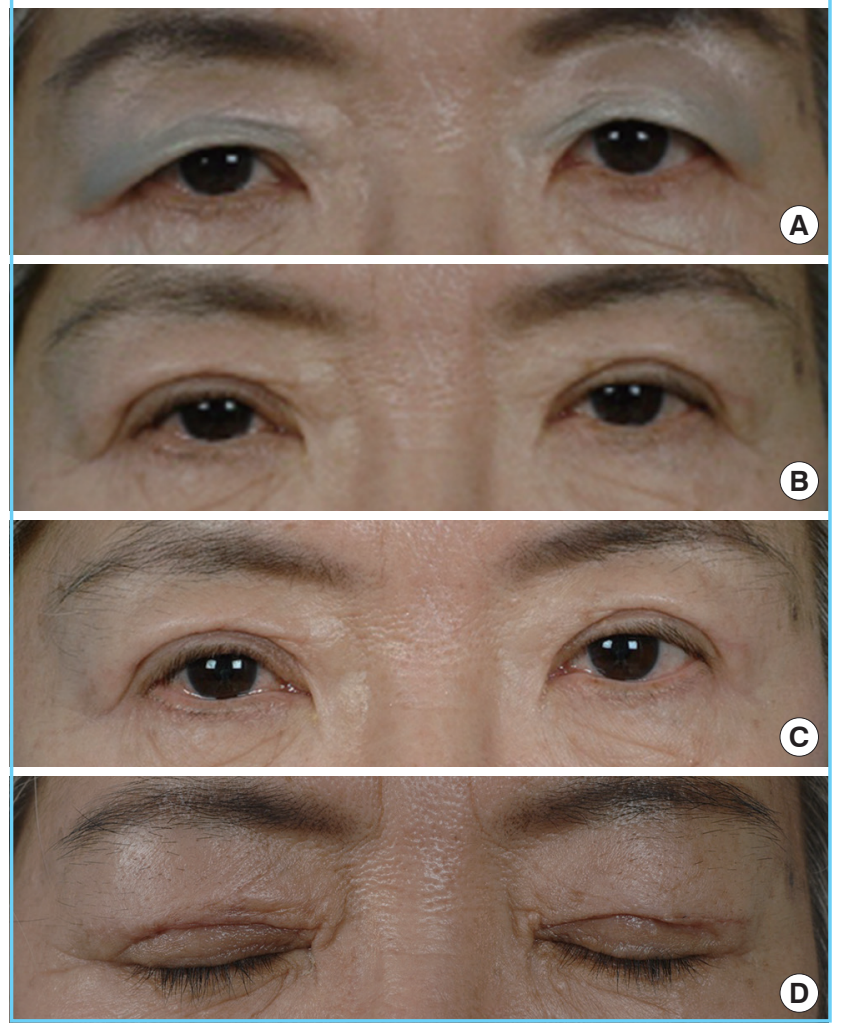

DISCUSSION

The first trial of suture double-eyelid blepharoplasty was performed by Mikamo in 1896 [4], and more than 32 modified surgical methods have been described in the Japanese literature over the past 90 years [5]. These methods can be divided into three groups, non-incisional (buried suture), partial incision, and fullincision techniques [2]. Non-incisional double-eyelid blepharoplasty has been gaining popularity in Korea, although incisional and partial incision techniques are still more common. Non-incisional blepharoplasty has a short recovery time, lacks visible scarring, minimizes postoperative bruising, and allows the possibility of a return to the preoperative shape if the patient wants to remove the double-eyelid in the future [6]. However, non-incisional double-eyelid operations are more appropriate for young patients, patients with minimal blepharochalasia and non-bulky upper eyelids, and patients needing revisional blepharoplasty due to lowering of their upper blepharoplasty line [5]. When the upper eyelid skin laxity is greater than 3-4 mm, or the upper eyelid is bulky enough such that resection of the orbicularis oculi muscle is needed, incisional blepharoplasty is the most com- monly used technique [2]. The incisional blepharoplasty method may require excision of the pretarsal orbicularis oculi muscle, soft tissue and orbital fat, form a stronger connection from the levator aponeurosis to the skin, and make a firm supratarsal fold. However, it leaves a noticeable scar on the line when patients close their eyes, and the larger insult to pretarsal tissue, blood, and lymphatic drainage causes long lasting swelling [7].

Some authors have explored modified methods to obtain advantages associated with buried suture techniques in doubleeyelid blepharoplasty. Cho and Byun [8] reported a new technique that combined the suture and incision methods. The levator aponeurosis was fixed to the tarsal plate through a partial skin incision, and a single continuous buried suture was placed. The modified technique reported in the present paper is also a combined method using non-incisional suturing and incisional double-eyelid blepharoplasty. However, in our new method, we fixed the blepharoplasty line first according to the non-incisional method, followed by excision of the upper eyelid skin and fat. Therefore, our method preserves part of the lymphatic circulation, decreasing postoperative bruising and treatment period.

Our method provides superior aesthetic results compared to other blepharoplasty techniques in elderly patients with the advantage of easily making accurate lines at the desired points. We have tried other techniques previously, and found that the nonincisional method has natural and satisfying aesthetic results. If we make the line first and then excise excessive skin using a different incision, we obtain excellent results in terms of naturallooking upper eyelid shapes and correction of blepharochalasia, thereby combining the advantages of suture and incisional methods. The removal of pretarsal tissue such as pretarsal fat and orbital fat can make the double fold firmer because it results in a wider contact area between the dermis and the tarsal plate. In addition to the removal of pretarsal fat, which enhances the durability of the double fold after non-incisional blepharoplasty, subsequent excision of the remaining skin provides the effects of incisional blepharoplasty and minimizes the likelihood of recurrence.

Our method is very reproducible due to the fact that the tarsodermal fixation is performed first. There is no damage to the capillary arcade, and therefore a more natural double fold develops due to preservation of the orbicularis oculi muscle. Dimpling at the fixation points was prevented due to differences between the points of the blepharoplasty line and skin excision line, so patients had greater satisfaction with their postoperative scarring.

In conclusion, we devised a new method of geriatric blepharoplasty in which tarsodermal fixation is first performed and then incisional blepharoplasty with fat repositioning is performed for the correction of sunken eyes. Our method has the following advantages: 1) ease of obtaining a natural-looking fold with sym- 
metry at the desired point; 2) reproducible methods that require short operation times; 3 ) fast postoperative recovery that results in a natural-appearing double-eyelid line; and 4) high patient satisfaction. Tarsodermal fixation performed before incision results in a natural-appearing double-eyelid line, even when patients have severe blepharochalasia, and the surgical time is similar to that of simple non-incisional blepharoplasty, with higher satisfaction and a decreased postoperative recovery time.

\section{REFERENCES}

1. Chen WP, Park JD. Asian upper lid blepharoplasty: an update on indications and technique. Facial Plast Surg 2013; 29:26-31.

2. Jinghe $Z$, Huifang $X$, Lihong $W$, et al. Three mini-incision double-eyelid blepharoplasty. Ann Plast Surg 2014;72:141-4.

3. Kim YK, Kwon JD, Oh KS. Double eyelid operation with three tiny incisions. J Korean Soc Plast Reconstr Surg 2000; 27:195-8.

4. Mizuno T. Two modified techniques to decrease complications of buried suture double-eyelid blepharoplasty. J Plast Reconstr Aesthet Surg 2013;66:e95-100.

5. Mikamo M. Mikamo's double-eyelid operation: the advent of Japanese aesthetic surgery. 1896. Plast Reconstr Surg 1997;99:664.

6. Liao WC, Tung TC, Tsai TR, et al. Celebrity arcade suture blepharoplasty for double eyelid. Aesthetic Plast Surg 2005; 29:540-5.

7. Chen WP. Suture ligation methods. In: Chen WP, editor. Asian blepharoplasty and the eyelid crease. Philadelphia: Butterworth Heinemann/Elsevier; 2006. p.39-50.

8. Cho BC, Byun JS. New technique combined with suture and incision method for creating a more physiologically natural double-eyelid. Plast Reconstr Surg 2010;125:324-31. 